where $\mu=n^{2} \alpha_{1}-m \alpha_{2}$. For a wedge airfoil with flat base (on which the unknown base pressure is assumed to be constant, though possibly time dependent) the normal force and pitching moment coefficients are

$C_{N}=\left(2 / M_{1}^{2}+\gamma \bar{C}_{p}\right)\left[2 A \alpha+(2 B \sigma+C) \alpha \cdot \sec \epsilon / a_{2}\right]$,

$C_{M}=\left(2 / M_{1}^{2}+\gamma \bar{C}_{p}\right) \sec ^{2} \epsilon\left[A(2 \sigma-1) \alpha+\left\{B \sigma(2 \sigma-1)+C\left(\sigma-\frac{2}{3}\right)\right\} \alpha^{\cdot} \sec \epsilon^{\prime} a_{2}\right]$,

where $\sigma=c \cos ^{2} \epsilon / c$.

The term in Eq. (16) proportional to $\alpha$ represents the aerodynamic damping moment for slow oscillations, which tends to stabilize if it is negative. The boundary of neutral stability as it depends upon pivot position and free-stream Mach number is shown in Fig. 2 for a wedge airfoil of $5^{\circ}$ semi-vertex angle. Also shown are the corresponding

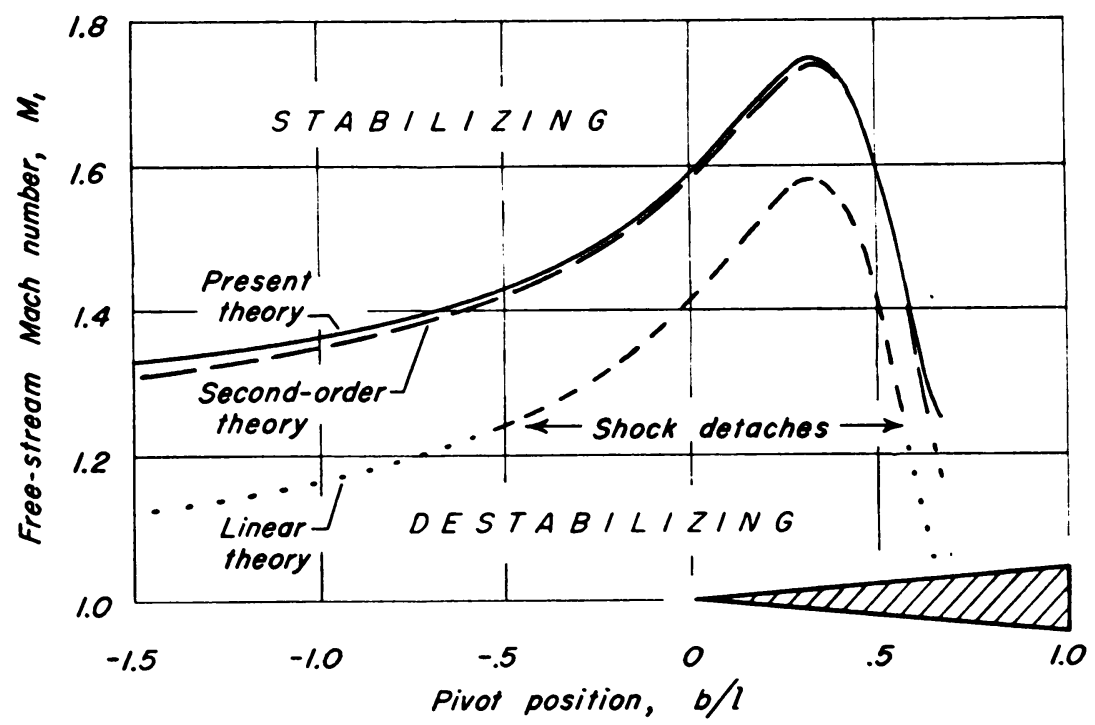

FIG. 2. Boundary of neutral stability for slowly oscillating wedge airfoil of $5^{\circ}$ semi-vertex angle.

results from linear and second-order theory ${ }^{4}$ (with which the present theory agrees when expanded in powers of $\epsilon$ ), which are applicable to any airfoil shape.

\title{
A NOTE ON SUBSONIC EDGES IN UNSTEADY SUPERSONIC FLOW*
}

\section{By JOHN W. MILES (University of California, Los Angeles)}

Summary. The pressure distribution due to the unsteady motion of a wing having a supersonic leading edge and a subsonic trailing edge is determined by applying a Lorentz transformation to the corresponding result for a rectangular wing. This result, valid as

${ }^{4}$ Milton D. Van Dyke, On second-order supersonic flow past a slowly oscillating airfoil, J. Aero. Sci. 20, 61 (1953).

*Received March 2, 1953. 
it stands for a subsonic leading edge, is then modified by the addition of an eigensolution in order to satisfy the Kutta condition.

1. Introduction. It was noted in an earlier paper [1] that the general solution for a rectangular wing in unsteady supersonic flow could be extended to a wing having a straight subsonic leading edge with the aid of the Lorentz transformation. It is the purpose of the present note to extend this result to a subsonic trailing edge. (The corresponding steady flow problem has been treated by Lagerstrom [2] and others.)

2. The potential equation. The notation of the present paper is in agreement with that of ref. 1, to which we refer by the prefix I ( ), where $($ ) denotes the equation number therein. In the interests of brevity, the following analysis is written as a continuation of $I$ and repeats equations and results stated therein only insofar as appears necessary to define the problem at hand.

The original coordinates (dimensionless, being referred to a characteristic length $l$ ) are the orthogonal Cartesian set $(x, y, z)$, where the free stream velocity $U$, is directed along $+x$. In these coordinates, the projection on $z=0$ of the wing to be considered is bounded by

$$
\begin{aligned}
& x+m \beta y>0 \\
& m \beta x+y \geqq 0 \\
& -1<m \leqq 0 \\
& \beta=\left(M^{2}-1\right)^{1 / 2}
\end{aligned}
$$

where $x+m \beta y=0$ defines a straight, supersonic leading edge and $m \beta x+y=0$ a straight, subsonic trailing edge. The definition of the leading edge is dictated by convenience (so $x^{\prime}=0$ there; $v . i$.) and may be varied at will, insofar as it remains supersonic.

We next introduce the coordinates $(\xi, \tau)$ by cascading Gallilean [I (2.3)] and modified Lorentz $[\mathrm{I}(2.4)]$ transformations as follows:

$$
\left(\begin{array}{l}
\xi \\
\tau
\end{array}\right)=\beta^{-1}\left(\begin{array}{rr}
1 & M \\
M & 1
\end{array}\right)\left(\begin{array}{rr}
1 & -M \\
0 & 1
\end{array}\right)\left(\begin{array}{c}
x \\
a t / l
\end{array}\right)
$$

where $t$ and $a$ represent true time and the sonic velocity, respectively. Finally, we introduce the oblique coordinates $\left(x^{\prime}, y^{\prime}\right)$ via the true Lorentz [I(8.1)] transformation

$$
\left(\begin{array}{l}
x^{\prime} \\
y^{\prime}
\end{array}\right)=\left(1-m^{2}\right)^{-1 / 2}\left(\begin{array}{cc}
1 & m \\
m & 1
\end{array}\right)\left(\begin{array}{l}
\xi \\
y
\end{array}\right)
$$

In these coordinates the leading and trailing edges are specified by $x^{\prime}=0$ and $y^{\prime}=0$, respectively. Then, taking $\left(x^{\prime}, y^{\prime}, z, \tau\right)$ as our working coordinates, the equation for the velocity potential, obtained by applying the Lorentz transformation to $I(2.6)$, reads

$$
\phi_{x^{\prime} x^{\prime}}-\phi_{\nu^{\prime} \nu^{\prime}}-\phi_{z z}=\phi_{\tau \tau}
$$


The corresponding equation for the pressure perturbation, obtained by applying (2.5) to I(2.12), may be written

$$
\begin{gathered}
p=-\rho_{0} a \beta^{-1} \chi \\
\chi=\chi\left(x^{\prime}, y^{\prime}, z, \tau\right)=\left(1-m^{2}\right)^{-1 / 2} M\left(\phi_{x^{\prime}}+m \phi_{\nu^{\prime}}\right)+\phi_{\tau}
\end{gathered}
$$

The integration of (2.8), subject to the boundary condition $\phi=0$ at the leading edge, yields,

$\phi_{-}=\left(1-m^{2}\right)^{1 / 2} M^{-1} \int_{0}^{z^{\prime}} \chi\left[\mu, y^{\prime}-m\left(x^{\prime}-\mu\right), \tau-\left(1-m^{2}\right)^{1 / 2} M^{-1}\left(x^{\prime}-\mu\right)\right] d \mu$

3. The boundary conditions. The wing platform (S), as it appears in the $(\xi, y)$ and $\left(x^{\prime}, y^{\prime}\right)$ coordinates, is shown in Fig. 1 . The wake aft of the trailing edge is designated

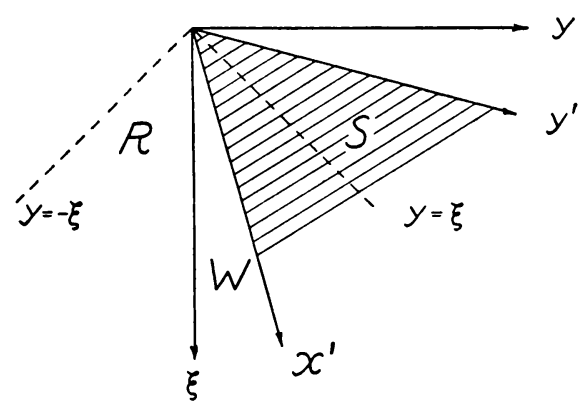

FIG. 1. The regions $\mathbf{S}, \mathbf{W}$ and $\mathbf{R}$, the various coordinate axes, and the Mach lines $y= \pm \xi$.

as $\mathbf{W}$ and the remainder of the plane $z=0$ as $\mathbf{R}$. The boundary conditions in $\mathbf{S}$ and $\mathbf{R}$ are as in $\mathrm{I}(2.7)$ and $\mathrm{I}(2.10)$, respectively, while the boundary condition in $\mathrm{W}$ is dictated by the requirement of continuity of pressure. Thus, we obtain

$$
\begin{array}{llll}
\phi_{z}=-w^{\prime}\left(x^{\prime}, y^{\prime}, \tau\right) & \text { on } & \mathbf{S}\left(x^{\prime}>0, y^{\prime}>0, z=0 \pm\right) \\
\chi=0 & \text { on } & \mathbf{W}\left(x^{\prime}>0, m x^{\prime} \leqq y^{\prime} \leqq 0, z=0\right) \\
\phi=0 & \text { on } & \mathbf{R}\left(x^{\prime}>0, y^{\prime}<m x^{\prime}, z=0\right)
\end{array}
$$

where $w^{\prime}$, the prescribed downwash, is now exhibited as a function of the oblique coordinates.

In the case of a non-trailing edge the region $\mathrm{W}$ would not exist, and (3.3) would hold for $y^{\prime} \leqq 0$. The corresponding solution for $\phi$, designated as $\phi^{(1)}$ in the following, and its derivatives $\phi_{x^{\prime}}$, and $\phi_{r}$ then would vanish as $y^{\prime 1 / 2}$ at the edge $y^{\prime}=0$, while $\phi_{y^{\prime}}$, would behave as $y^{\prime-1 / 2}$ there. Accordingly, for $m>0 \chi$ would exhibit the expected singularity associated with a subsonic leading edge ( $c f$. ref. 2 ). On the other hand, for $m<0$ an eigensolution, $\phi^{(2)}$, can be determined to cancel this singularity, thereby satisfying the Kutta condition $\left(\chi=0\right.$ at $\left.y^{\prime}=0\right)$. Accordingly, we write

$$
\phi=\phi^{(1)}+\phi^{(2)}
$$

and formulate two, subsidiary boundary value problems. 
As indicated in the foregoing, $\phi^{(1)}$ may be assumed to satisfy the boundary conditions

$$
\begin{array}{lll}
\phi_{z}^{(1)}=-w^{\prime} & \text { on } & \mathbf{S}\left(x^{\prime}>0, y^{\prime}>0, z=0 \pm\right) \\
\phi^{(1)}=0 & \text { on } & \mathbf{W}+\mathbf{R}\left(x^{\prime}>0, y^{\prime} \leqq 0, z=0\right)
\end{array}
$$

It follows that $\phi_{i}^{(2)}$ and, therefore, $\chi_{2}^{(2)}$ must vanish in S in order to satisfy (3.1). Moreover, since $\phi^{(2)}$ must vanish in R, so also must $\chi^{(2)}$. Hence, we write

$$
\begin{array}{lll}
\chi_{z}^{(2)}=0 & \text { on } & \mathbf{S} \\
\chi^{(2)}=0 & \text { on } & \mathbf{W}+\mathbf{R}
\end{array}
$$

To complete the formulation, we remark that $\chi$ also satisfies the differential equation (2.6).

As will be shown below, the explicit determination of $\phi^{(2)}$ is not required insofar as $\chi$ alone is required. However, given $\chi^{(2)}, \phi^{(2)}$ may be determined from (2.9).

4. Solution. The boundary value problem of determining $\phi^{(1)}$ when $w^{\prime}$ is prescribed is now identical with the rectangular wing problem solved in I. A convenient form of the solution for our present purpose is obtained by posing the harmonic time dependence $\exp \left(i_{\kappa} \tau\right)$, substituting $x^{\prime}$ and $y^{\prime}$ for $\xi$ and $y$ in I(4.5) and introducing the trigonometric change of variable $\eta=y^{\prime}+\left(x^{\prime}-\mu\right) \cos \theta \cos \lambda$, whence we have

$$
\begin{aligned}
& \phi^{(1)}\left(x^{\prime}, y^{\prime}, 0+, \tau\right)=\int_{0}^{x^{\prime}} J_{0}\left[\kappa\left(x^{\prime}-\mu\right)\right] w^{\prime}\left(\mu, y^{\prime}, \tau\right) d \mu \\
& -\frac{1}{\pi} \int_{0}^{x^{\prime}} d \mu \int_{0}^{\pi / 2} J_{0}\left[\kappa\left(x^{\prime}-\mu\right) \sin \theta\right] d \theta \\
& \cdot \frac{\partial}{\partial \theta} \int_{0}^{2 \sin ^{-3}\left[y^{\prime} /\left(x^{\prime}-\mu\right) \cos 0\right]^{2 / 3}} w^{\prime}\left[\mu, y^{\prime}+\left(x^{\prime}-\mu\right) \cos \theta \cos \lambda, \tau\right] d \lambda
\end{aligned}
$$

where the arc-sine is to be replaced by $\pi / 2$ when its argument exceeds unity. In the following, we refer to this solution in the symbolic form

$$
\phi^{(1)}\left(x^{\prime}, y^{\prime}, 0+, \tau\right)=\phi^{(1)}\left\{w^{\prime}\left(x^{\prime}, y^{\prime}, \tau\right)\right\}
$$

To obtain $\chi^{(2)}$, we remark that, since any derivative of $\phi$ also satisfies $(2.6), \phi_{y^{\prime}}^{(1)}\left\{w^{\prime}\right\}$ represents a solution satisfying the boundary conditions (3.5) and (3.6) provided that $w^{\prime}$ is replaced by $w_{\nu}^{\prime}$, therein. Moreover, $\phi^{(1)}\left\{w_{\nu}^{\prime}\right\}$ satisfies the same differential equation and boundary condition as $\phi_{y^{\prime}}^{(1)}$ but differs therefrom in vanishing at $y^{\prime}=0$, whereas $\phi_{\nu^{\prime}}^{(1)}$ is singular as $y^{\prime-1 / 2}$ in this neighborhood. It follows that $\phi_{y^{\prime}}^{(1)}\left\{w^{\prime}\right\}-\phi^{(1)}\left\{w_{y^{\prime}}^{\prime}\right\}$ represents a solution to the differential equation (2.6) satisfying the homogeneous boundary conditions (3.7) and (3.8) and exhibiting the desired singularity at $y^{\prime}=0$. Accordingly, the required solution for $\chi^{(2)}$ that cancels the singularity in $\chi^{(1)}$ at $y^{\prime}=0$ is given by

$$
\chi^{(2)}=-m\left(1-m^{2}\right)^{-1 / 2} M\left[\phi_{\nu^{\prime}}^{(1)}\left\{u^{\prime}\right\}-\phi^{(1)}\left\{w_{\nu^{\prime}}^{\prime}\right\}\right]
$$


Substituting from (4.1), we have

$$
\begin{aligned}
\chi^{(2)}=m(1- & \left.\left.m^{2}\right)^{-1 / 2} M y^{\prime-1 / 2} \pi^{-1} \int_{0}^{x^{\prime}} d \mu \int_{0}^{00 s^{-1}\left|y^{\prime} /\left(x^{\prime}-\mu\right)\right|} J_{0}\left[\kappa\left(x^{\prime}-\mu\right) \sin \theta\right)\right] \\
& \cdot \frac{\partial}{\partial \theta}\left\{\left[\left(x^{\prime}-\mu\right) \cos \theta-y^{\prime}\right]^{-1 / 2} w^{\prime}\left[\mu,\left(x^{\prime}-\mu\right) \cos \theta-y^{\prime}, \tau\right]\right\} d \theta
\end{aligned}
$$

The final solution for the pressure distribution on the upper surface of the wing, obtained by substituting $\phi^{(1)}$ in (2.8) and adding $\chi^{(2)}$ from (4.3), is given by

$$
\chi\left(x^{\prime}, y^{\prime}, 0+, \tau\right)=\left(1-m^{2}\right)^{-1 / 2} M\left[\phi_{x^{\prime}}^{(1)}\left\{w^{\prime}\right\}+m \phi^{(1)}\left\{w_{\nu^{\prime}}^{\prime}\right\}\right]+\phi_{\tau}^{(1)}\left\{w^{\prime}\right\}
$$

We remark that the results (4.3) and (4.5) are not restricted to harmonic time dependence, since they are valid for all frequencies.

In the case of a subsonic leading edge $(m \geq 0)$, it would be necessary only to replace $\phi^{(1)}\left\{w_{y^{\prime}}^{\prime}\right\}$ by $\phi_{y^{\prime}}^{(1)}\left\{w^{\prime}\right\}$ in (4.3).

\title{
REFERENCES
}

1. J. W. Miles, A general solution for the reclangular airfoil in supersonic flow, Q. Appl. Math., 11, 1-8 (1952).

2. P. A. Lagerstrom, Linearized supersonic theory of conical wings, J. P. L. Prog. Rep. 4-36 Pasadena, (1947); reprinted as NACA TN 1685 (1948), 90-98.

\section{NOTE ON THE MEAN SQUARE VALUE OF INTEGRALS IN THE STATISTICAL THEORY OF TURBULENCE*}

\author{
By C. C. LIN (Massachusetts Institute of Technology)
}

1. In the statistical theory of homogeneous isotropic turbulence, it is sometimes of interest to evaluate the mean square value of certain integrals, such as the pressure fluctuation over a sphere. The purpose of the present note is to give such an evaluation for integrals over a sphere and for similar integrals over spaces of other dimensions. The analysis shows that the final answer can be interpreted in terms of dimensional arguments; provided the length scale used is the geometrical mean of the scale of turbulence and the linear scale of the region over which the integral is taken. The results could be applied to the problem of the noise generated by turbulence.

2. Consider, for definiteness, the pressure fluctuation over the surface of a sphere. Extension to the study of other quantities can be easily made. Let the integral be denoted by

$$
I=\int p d S
$$

where $p$ is the pressure fluctuation at a point $P$, and the surface integral is extended over a sphere of radius $a$. We may also write

$$
I=\int p^{\prime} d S^{\prime}
$$

"Received April 20, 1953. 